\title{
How Do White LEDs' Spectra Affect the Fresh Weight of Lettuce Grown under Artificial Lighting in a Plant Factory?-A Statistical Approach
}

\author{
Na Lu ${ }^{1}$, Suthisak Saengtharatip², Michiko Takagaki1,2*, Atsushi Maruyama², Masao Kikuchi1 \\ ${ }^{1}$ Center for Environment, Health and Field Sciences, Chiba University, Chiba, Japan \\ ${ }^{2}$ Graduate School of Horticulture, Chiba University, Chiba, Japan \\ Email: na.lu@chiba-u.jp, unatic_asylumz@yahoo.com, ^mtgaki@faculty.chiba-u.jp, a.maruyama@faculty.chiba-u.jp, \\ m.kikuchi@faculty.chiba-u.jp
}

How to cite this paper: Lu, N., Saengtharatip, S., Takagaki, M., Maruyama, A. and Kikuchi, M. (2019) How Do White LEDs' Spectra Affect the Fresh Weight of Lettuce Grown under Artificial Lighting in a Plant Factory?-A Statistical Approach. Agricultural Sciences, 10, 957-974.

https://doi.org/10.4236/as.2019.107073

Received: June 17, 2019

Accepted: July 22, 2019

Published: July 25, 2019

Copyright (c) 2019 by author(s) and Scientific Research Publishing Inc. This work is licensed under the Creative Commons Attribution International License (CC BY 4.0).

http://creativecommons.org/licenses/by/4.0/

\begin{abstract}
Monochromatic light-emitting diode lamps (LEDs), emitting red and blue lights, revolutionized crop production in closed-system plant factories with artificial lighting in the early 1990s. The LED industry developed broad-spectrum white-LEDs by 2010, and many types of white-LEDs for home and office uses are now available for plant factory entrepreneurs. This paper tries to clarify whether these white-LEDs can be used as effective light sources in plant factories by examining what types of spectrum distribution are better suited for plant production. An experiment was conducted using seven LEDs, of which six were white-LEDs, to compare the performance in producing lettuce, and the results were compared with recent studies that used white-LEDs for growing lettuce under closed-system production conditions. Results showed that broad-spectrum white-LEDs performed significantly better than narrow-spectrum LEDs. Among lights in conventional color bands, red and blue lights give critical effects on plant growth, not in isolation but in combination; not too "cool" white LEDs perform better. Green and far-red lights also have some positive effects. Altogether, for a given light intensity, broad-spectrum white LEDs outperform narrow-spectrum LEDs. It is found that the spectrum distribution for white-LEDs to attain high productivity in lettuce production is such that the percentage share of photon flux density by conventional color band falls in the following ranges: $0 \%<$ blue $<30 \%, 0 \%<$ green $<50 \%, 30 \%<$ red $<70 \%$, and $0 \%<$ far-red $<20 \%$.
\end{abstract}

\section{Keywords}

Broad Spectrum LED, Lettuce Yield, Narrow Spectrum LED, Response Function, 
Spectrum Distribution

\section{Introduction}

Vegetable production in closed-system plant factories with artificial lighting, also called vertical farming [1], has seen great progress since light-emitting diodes (LEDs) were introduced in the 1980s to replace conventional light sources such as fluorescent lamps (FLs) [2] [3]. The use of LEDs in plant production has followed the technological innovations in the LED industry. High-efficiency red LEDs (R-LEDs) had been developed in the 1980s, followed by the development of high-efficiency blue LEDs (B-LEDs) in the early 1990s [4]. It was found by the early 2000s that the use of monochromatic R-LEDs, supplemented by B-LEDs, attained the plant productivity as high as under sunlight [5], and these monochromatic narrow spectrum LEDs or red + blue LEDs (RB-LEDs) have dominated in research in this field as well as in commercial plant factory production [6].

The LED industry had developed by the late-1990s high-efficiency LEDs covering the entire visible spectrum from blue to red as well as the phosphor technology to create white LEDs (W-LEDs) by converting a monochromatic narrowband light from a LED chip to a broadband white light, and the commercial supply of W-LEDs began in 2010 [4]. Since then, the use of W-LEDs has rapidly spread to all spheres of human life to replace conventional lights. In the last four decades since its advent, the price of LEDs has declined geometrically by one-tenth every decade [4]. Large prospective demands yet forthcoming would continue to reduce the price of $\mathrm{W}$-LEDs at even higher speed.

White light consists of blue, green, and red lights, and there are three major methods to make a LED lamp that emits these lights. The first method is to incorporate red, blue and green LED chips into one LED lamp, the second one is to convert a part of blue light from a B-LED into red and green using a phosphor, and the third method is to add a red-LED chip to a white LED lamp made by the second method for enhancing red light. All are white LEDs, but in this paper, we distinguish the first type as RGB-LED, the second type as W-LED, and the third type as RW-LED, and denote LEDs including all the three types as whitish LEDs. Because of its efficiency, simplicity, and the requirement of only one power supply for one chip, the second chip-plus-phosphor approach has attained a pervasive commercial success [4], and whitish LEDs, which are flooding in the market at ever declining prices, are W-LEDs.

The typical spectrum distribution of the first-generation W-LED has a sharp peak at around $450 \mathrm{~nm}$ in the blue band and the summit, or hilltop, at around $560 \mathrm{~nm}$ in a phosphor-converted hilly broad band stretching from the green to the red, and to the far-red bands. In 20 years since this proto-type was made, various types of W-LEDs with different spectral distributions over these color bands have been developed within the distribution pattern of a sharp-peak in the 
blue-band and a broad-hilly-band from the green- to red-, and to far-red-bands. In this study, following Cope et al. (2014) [7], W-LEDs are classified into three types: cool-white (CW) with a higher share of photon-flux density (PFD) in the blue-band; warm-white (WW) with a lower share in the blue-band and a higher share in the red-band; and neutral-white (NW), between CW and WW, with a higher share in the green-band.

More than a decade later after its advent, various types of W-LEDs with different spectra are now available, but there is virtually no guideline on what types of spectrum characteristics W-LEDs should have to be introduced by plant-factory entrepreneurs. Considering the importance of the roles to be played by W-LEDs in plant factories in near future, it is vitally important for vegetable production in plant factories to assess how the performance of W-LEDs in growing plants is compared to that of conventional light sources including RB-LEDs, and what types of W-LEDs with specific spectral distributions are better suited for vegetable production.

Studies on the effects of W-LEDs on plant growth, which began in the early 2010s, have been sharply increasing in the last few years. Even if limited to lettuce, on which this paper focuses, many studies have recently published, and many of them have demonstrated that the effects of broad-spectrum W-LEDs on lettuce growth are as good as, or even better than, those of narrow-spectrum R-LED, B-LED, and RB-LED [7] [8] [9] [10]. However, the results of the studies varied greatly, and some reported opposite results [11] [12]. Conflicting results were also reported among whitish-LEDs, i.e., among RGB-LED, RW-LED and W-LED [13] [14] [15]. Moreover, plant-growth performance varied considerably among broad-spectrum W-LEDs [7]. The diversity of the results among these studies is very large. More studies are urgently required to obtain comprehensive understanding about how W-LEDs work on plant growth.

The purpose of this study is to explore how the performance of whitish-LEDs is compared among them as well as with that of RB-LEDs which have been used as a major light source in plant factories. An experiment was conducted in which lettuce was grown in a commercially operated plant factory under artificial lighting supplied by seven LEDs with different spectrum patterns to examine how different spectrum distributions affect the growth of lettuce by using data on the photon-flux density (PFD) of different wavelength bands, and how far our observations can be generalized is examined by comparing the results of our experiment with those of past studies which used whitish-LEDs for growing lettuce under closed-system conditions.

\section{Materials and Methods}

\subsection{Materials}

Plant factory, plant materials, growth conditions, and sampling: Leaf lettuce was grown in a closed-system commercially operated plant factory with ar- 
tificial light of $400 \mathrm{~m}^{2}$ where all environmental conditions were controlled. Lettuce seeds (Lactuca sativa L., cv. Frill Ice; Yukijirushi Seed Co., Hokkaido, Japan) were sown in urethane cubes (W $2.3 \mathrm{~cm} \times \mathrm{D} 2.3 \mathrm{~cm} \times \mathrm{H} 2.7 \mathrm{~cm}$ ) soaked with tap water and placed in the dark at $20^{\circ} \mathrm{C}$ for $48 \mathrm{~h}$ for germination. Seedlings were raised hydroponically under CW-FLs (Panasonic FHF32EX-N-H) at a PFD of $150 \mu \mathrm{mol} \cdot \mathrm{m}^{-2} \cdot \mathrm{s}^{-1}$ for $16 \mathrm{~h} \cdot \mathrm{d}^{-1}$. At 12 days after seeding (DAS), uniform-sized plantlets were transplanted into polystyrene panels $\left(144.4\right.$ plants $\left.\cdot \mathrm{m}^{-2}\right)$ and cultivated under seven different types of LEDs at the light intensity of PFD $=200 \pm 10$ $\mu \mathrm{mol} \cdot \mathrm{m}^{-2} \cdot \mathrm{s}^{-1}$ on the surface of the cultivation panel, as measured by a light sensor (Licor 250A; Li-cor Inc., Lincoln, NE, USA), for $14 \mathrm{~h} \cdot \mathrm{d}^{-1}$. At $23 \mathrm{DAS}$, the plantlets were spaced (33.3 plants. $\left.\mathrm{m}^{-2}\right)$ and cultivated in the same light condition until harvest at 35 DAS. Panels with plants were placed in a shelf of seven layers, each LED taking a layer. The dimension of one plant growing layer that was lighted by a LED was $1050 \mathrm{~cm} \times 120 \mathrm{~cm} \times 30 \mathrm{~cm}(\mathrm{~L} \times \mathrm{W} \times \mathrm{H}) \cdot \mathrm{CO}_{2}$ concentration was controlled at $1200 \mu \mathrm{mol} \cdot \mathrm{m}^{-2} \cdot \mathrm{s}^{-1}$ throughout the growing period. The seven LEDs consisted of four W-LEDs, two RW-LEDs, and one RB-LED. The plants from all treatments (LEDs) were hydroponically grown via the deep flow technique. After harvesting from the cultivation area, shoot $F W$ was measured. The growth conditions throughout the experiment are summarized in Table 1.

The experiment was conducted in two rounds under the same setting and conditions, and six and nine plants were sampled in each round for measurement. Since the growing conditions were controlled across all the treatments, the 15 sampled plants for each treatment were treated as the 15 replications of the experiment. Our experimental design assumed that systematic variation arose only from possible differences in seeds and that all other sources of variation distributed independently and randomly around the mean 0 . The number of plants sampled increased from six to nine from the first to the second rounds in order to attain the power of detection at $80 \%$ or higher in the multiple-comparison

Table 1. Growth conditions of lettuce harvested at 35 days after sowing.

\begin{tabular}{ccccc}
\hline \multirow{2}{*}{ Condition } & \multicolumn{2}{c}{ Seedling $(12$ days $)$} & \multicolumn{2}{c}{ After transplanted (23 days) } \\
\cline { 2 - 5 } & Light & Dark & Light & Dark \\
\hline Air temperature $\left({ }^{\circ} \mathrm{C}\right)$ & 25 & 20 & 25 & 20 \\
Relative humidity $(\%)$ & 45 & 65 & 45 & 65 \\
$\mathrm{CO}_{2}$ concentration $\left(\mu \mathrm{mol} \cdot \mathrm{mol}^{-1}\right)^{\mathrm{a}}$ & 1200 & 1200 & 1200 & 1200 \\
Light source & Fluorescent lamp & - & LED lamp & - \\
Photoperiod $(\mathrm{h})$ & 16 & 8 & 14 & 10 \\
PFD $\left(\mu \mathrm{mol} \cdot \mathrm{m}^{-2} \cdot \mathrm{s}^{-1}\right)$ & 150 & - & 200 & - \\
Nutrient solution & $\mathrm{b}$ & $\mathrm{EC}=1.8 \mathrm{dS} \cdot \mathrm{m}^{-1}$ & EC $=2.4 \mathrm{dS} \cdot \mathrm{m}^{-1}$ \\
\hline
\end{tabular}

a) $\mathrm{CO}_{2}$ was controlled at one level throughout light and dark periods, because the experiment was conducted in a commercially operating plant factory side by side with other plant production. b) EC stands for Electrical Conductivity. For more details, see Saengtharatip et al. (2018) [28]. 
test of mean differences [16]. To simplify the analysis and clarify the effects of different spectra on plant growth, i.e., the result of photosynthesis activities, this paper concentrates its attention on shoot fresh weight $(F W)$.

Spectrum measurement and wavelength bands: The spectra of the seven LEDs were measured by a spectroradiometer (Lighting Passport, AsenseTek, Taiwan) over wavelengths ranging from $380 \mathrm{~nm}$ to $780 \mathrm{~nm}$, which is divided, following the convention in the research of this field, into five color bands: violet $(380-399 \mathrm{~nm})$, blue $(400-499 \mathrm{~nm})$, green $(500-599 \mathrm{~nm})$, red $(600-699 \mathrm{~nm})$, and far-red (700 - $780 \mathrm{~nm}$ ) denoted as V-, B-, G-, R-, and FR-bands, respectively. In addition to the conventional color-band demarcation, another band demarcation with narrower ranges of either $50 \mathrm{~nm}$ or $75 \mathrm{~nm}$ was adopted for the wavelengths between $425-700 \mathrm{~nm}$ to examine the sensitivity of plant growth to light of narrower color bands; 425 - $474 \mathrm{~nm}\left(\mathrm{~B}^{\prime}\right), 475$ - $524 \mathrm{~nm}$ (cyan = C), 525 $574 \mathrm{~nm}\left(G^{\prime}\right), 575-624 \mathrm{~nm}$ (yellow = Y), $625-699 \mathrm{~nm}\left(\mathrm{R}^{\prime}\right)$. This band demarcation was based on the inspection of the differences in the spectrum distribution over the entire range of PFD of the seven LEDs, but was incidentally the same as adopted by Kong et al. (2015) [13], except for the B'-band which was 400 - 475 $\mathrm{nm}$ in their study.

\subsection{Methods}

Four analytical methods were adopted to examine the relationship between lettuce $F W$ and LEDs' spectrum characteristics.

First, ANOVA. Since the experimental design of this study was one-way, ANOVA was applied as a means to make the multiple comparisons of means among the seven LEDs.

Second, graphical analysis. The distributional pattern of the spectrum in terms of percentage share of photon flux density (\%PFD) per nm over the range of 380 - $780 \mathrm{~nm}$ was compared for the seven LEDs vis-à-vis their rank in $F W$ productivity to identify the spectrum composition associated with high lettuce productivity.

Third, to examine how lights of different color bands were critically associated with $F W$, response functions that statistically described how $F W$ responded to different levels of PFD by color band were estimated. The basic form of the response function is

$$
F W_{i}=a+b X_{i j}+u_{i j},
$$

where $F W$ is fresh weight of lettuce $\left(\mathrm{kg} \cdot \mathrm{m}^{-2}\right), X_{i j}$ is the percentage share of PFD (\%PFD) in the entire spectrum of $j$-th color band $(j=1,2, \ldots, 5)$ of $i$-th $\operatorname{LED}(i=1,2, \ldots, 7), u_{i j}$ is a random error.

Three response patterns were found as the $F W$ s response to \% PFD within each of color bands: monotonically increases or decreases without any maximum with respect to \%PFD, or first increases, reaches the maximum, and then decreases (inverted-V shape distribution). For the last response pattern, $X_{i j}$ in 
Equation (1) is defined as the absolute value of $\left(X_{j}^{*}-X_{i j}\right)$, where $X_{j}^{*}$ is the $\%$ PFD in the $\mathrm{j}$-th color band which gives the maximum $F W$; in other words, the response function for the case in which the response pattern is subject to an inverted-V distribution measures how $F W$ responds to the distance from the best \%PFD that gives the best $F W$.

In estimating the response function by applying the least-squares method, three functional forms were tried out: i) linear, ii) semi-log linear ( $\log$ for $X_{i j}$ ), and iii) double-log linear, and the best-fitted form in terms of the coefficient of determination $\left(R^{2}\right)$ was searched. This estimation was made using the means over 15 replications for each of the seven LEDs, which means the number of observations $(n)=7$.

The fourth analytical method, which will be adopted in the discussion section, was the comparison of the results of our experiment with those of past studies that reported the yield performance of lettuce grown under closed-system conditions using whitish LEDs.

\section{Results}

\subsection{Yield Performance}

Lettuce yields, i.e., shoot fresh weight $(F W)$, ranged from $3.26 \mathrm{~kg} \cdot \mathrm{m}^{-2}(97.9$ g.plant ${ }^{-1}$ : achieved by LED\#1) to $1.92 \mathrm{~kg} \cdot \mathrm{m}^{-2}\left(57.7 \mathrm{~g} \cdot\right.$ plant $^{-1}$ : achieved by LED\#7), with the mean yield of $2.55 \mathrm{~kg} \cdot \mathrm{m}^{-2}$ (76.6 g.plant ${ }^{-1}$ ) (Table 2). The seven LEDs were numbered from \#1 to \#7 according to their order of yield performance, and multiple comparisons of yields sorted the seven LEDs into three productivity groups, i.e. top, middle and bottom, except for LED\#5 and LED\#6 which were not statistically distinguishable.

\subsection{Spectral Characteristics}

\section{PFD by wavelength}

The spectral patterns of the seven LEDs were markedly different (Figure 1). LED\#7 was an RB-LED and the rest were whitish-LEDs. Among whitish LEDs, LED\#4 and LED\#5 were RW-LEDs and LED\#1, LED\#2, LED\#3, and LED\#6 were W-LEDs, of which LED\#1, LED\#2, and LED\#3 were WW-LEDs, and LED\#6 was

Table 2. Shoot fresh weight $(F W)$ of lettuce grown for 35 days with LED, for seven LEDs tested ${ }^{\text {a }}$

\begin{tabular}{ccccccccc}
\hline & LED\#1 & LED\#2 & LED\#3 & LED\#4 & LED\#5 & LED\#6 & LED\#7 \\
\cline { 2 - 6 } & W-LED (WW) & W-LED (WW) & W-LED (WW) & RW-LED & RW-LED & W-LED (NW) & RB-LED & Mean \\
\hline$F W\left(\mathrm{~kg} \cdot \mathrm{m}^{-2}\right)$ & 3.26 & 3.21 & 2.62 & 2.40 & 2.35 & 2.12 & 1.92 & 2.55 \\
sd $\left(\mathrm{kg} \cdot \mathrm{m}^{-2}\right)$ & 0.39 & 0.35 & 0.20 & 0.31 & 0.21 & 0.27 & 0.31 & 0.56 \\
$F W\left(\mathrm{~g}^{-}\right.$plant $\left.^{-1}\right)$ & 97.9 & 96.2 & 78.6 & 71.9 & 70.6 & 63.5 & 57.7 & 76.6 \\
Multiple comparison $^{\mathrm{b}}$ & $\mathrm{a}$ & $\mathrm{a}$ & $\mathrm{b}$ & $\mathrm{b}$ & $\mathrm{bc}$ & $\mathrm{cd}$ & $\mathrm{d}$ & \\
\hline
\end{tabular}

a) Seven LEDs tested are numbered according to the ranking of lettuce shoot $F W$. $F W$ of each LED is the mean of 15 samples. b) Multiple comparison by Tukey method ( $\mathrm{p}<0.01$ ). For LEDs followed by the same alphabet, $F W$ is not statistically different. 


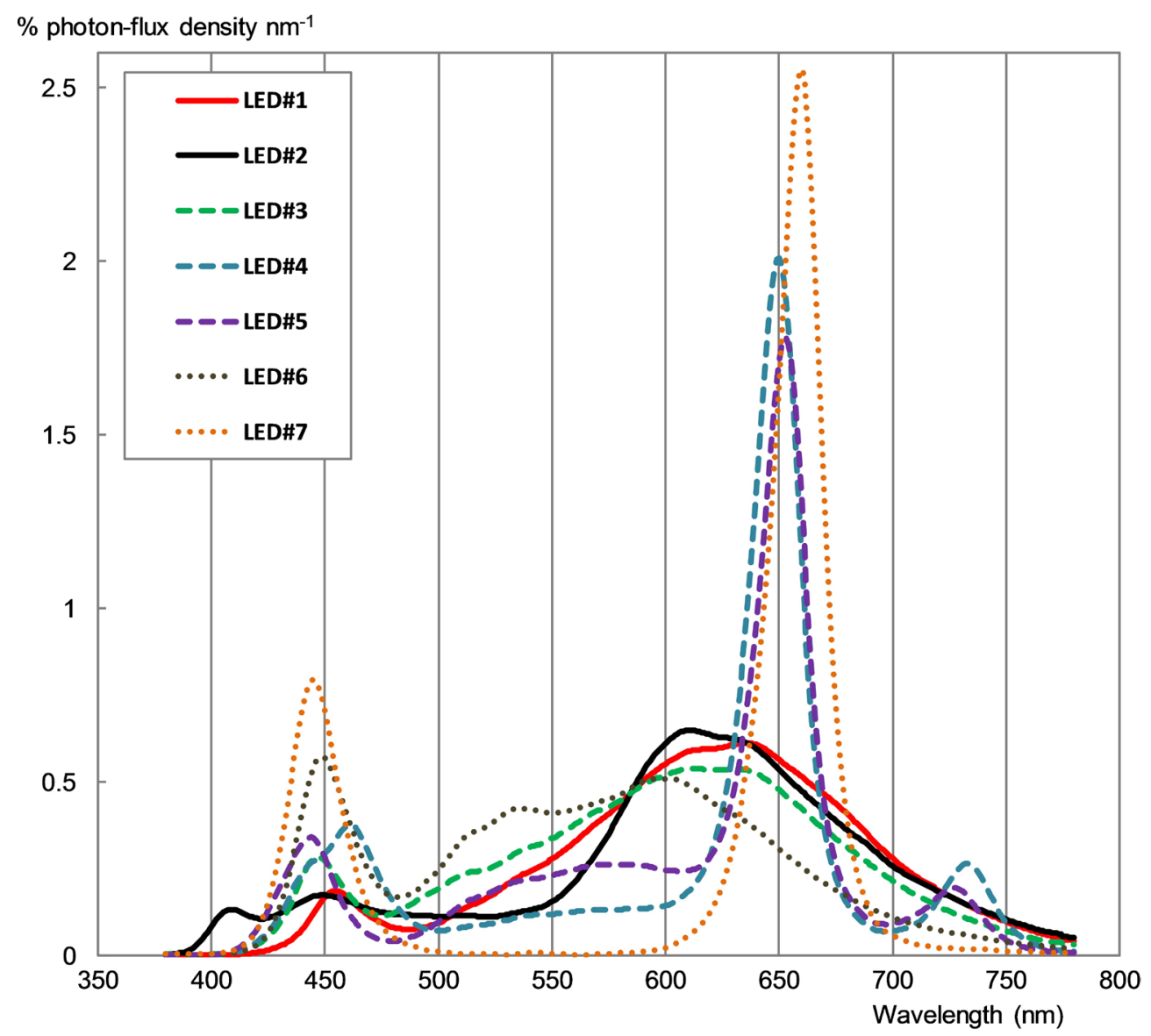

Figure 1. Percentage distribution of photon-flux density (\%PFD) in the total PFD in the entire wavelength range measured of seven LEDs tested at each wavelength (measured by $1 \mathrm{~nm}$ ) from 380 $\mathrm{nm}$ to $780 \mathrm{~nm}$. LEDs are numbered from \#1 to \#7 according to the order of fresh-weight productivity from the top to the bottom.

a NW-LED. The multiple comparison of the lettuce yields revealed that two WW-LEDs attained the best yield performance, followed by another WW-LED and two RW-LEDs, that the performance of RB-LED was poorest, and that the NW-LED performed as poor as the RB-LED (Table 2). These results, coupled with inspection of the distribution of PFDs over the entire wavelength range (Figure 1), yielded the following observations:

Observation I: Whitish LEDs generally performed better than the narrow-spectrum high-peak RB-LED.

Observation II: Among whitish LEDs, the broad-spectrum, low-peak W-LEDs of warm type performed better than the broad-spectrum, high-peak RW-LEDs and the W-LED of cooler type.

\section{Effects of light by color band}

Among the five conventional color bands, light in V-band was negligible for all the LEDs tested, at most $0.5 \%$ and $0.1 \%$ on average in terms of the percentage of photon flux density (\%PFD), and, in spite of its positive, significant correlation with lettuce $F W$, no meaningful response pattern was detected (Table 3 ). In 
Table 3. The percentage shares of photon flux density (\%PFD) by wavelength band in the total PFD in the entire wavelength range measured $(380-780 \mathrm{~nm})$ and their simple correlation coefficients with the fresh weight $(\mathrm{n}=105)$.

\begin{tabular}{|c|c|c|c|c|c|c|c|c|c|c|}
\hline & & $\begin{array}{l}\text { LED\# } 1 \\
\text { (WW) }\end{array}$ & $\begin{array}{l}\text { LED\#2 } \\
(\mathrm{WW})\end{array}$ & $\begin{array}{l}\text { LED\#3 } \\
\text { (WW) }\end{array}$ & $\begin{array}{l}\text { LED\#4 } \\
\text { (RW) }\end{array}$ & $\begin{array}{l}\text { LED\#5 } \\
\text { (RW) }\end{array}$ & $\begin{array}{l}\text { LED\#6 } \\
(\mathrm{NW})\end{array}$ & $\begin{array}{l}\text { LED\#7 } \\
\text { (RB) }\end{array}$ & \multicolumn{2}{|c|}{$\begin{array}{c}\mathrm{R} \\
\text { with } \mathrm{FW}^{\mathrm{c}}\end{array}$} \\
\hline \multicolumn{11}{|l|}{ [A] \% PFDa: } \\
\hline Violet (V) & $380-399 \mathrm{~nm}$ & 0.0 & 0.5 & 0.1 & 0.0 & 0.0 & 0.1 & 0.1 & 0.40 & ** \\
\hline Blue (B) & $400-499 \mathrm{~nm}$ & 7.7 & 13.3 & 13.1 & 16.5 & 12.3 & 22.7 & 23.7 & -0.71 & ** \\
\hline Green (G) & $500-599 \mathrm{~nm}$ & 29.8 & 24.3 & 34.9 & 11.5 & 21.8 & 41.5 & 0.8 & 0.29 & ** \\
\hline $\operatorname{Red}(\mathrm{R})$ & $600-699 \mathrm{~nm}$ & 51.4 & 50.8 & 43.7 & 62.7 & 58.7 & 31.2 & 73.8 & -0.19 & * \\
\hline Far-red (FR) & $700-780 \mathrm{~nm}$ & 11.1 & 11.1 & 8.3 & 9.3 & 7.2 & 4.5 & 1.7 & 0.77 & ** \\
\hline Total & & 100.0 & 100.0 & 100.0 & 100.0 & 100.0 & 100.0 & 100.0 & & \\
\hline \multicolumn{11}{|l|}{ [B] \% PFD } \\
\hline Blue' (B') & $425-474 \mathrm{~nm}$ & 5.5 & 7.4 & 8.9 & 13.0 & 9.9 & 17.4 & 21.8 & -0.76 & ** \\
\hline Cyan (C) & $475-524 \mathrm{~nm}$ & 5.5 & 5.8 & 9.5 & 5.0 & 5.2 & 13.1 & 1.1 & 0.00 & \\
\hline Green’ (G’) & $525-574 \mathrm{~nm}$ & 14.3 & 9.1 & 17.2 & 6.0 & 11.6 & 21.1 & 0.3 & 0.18 & \\
\hline Yellow (Y) & $575-624 \mathrm{~nm}$ & 26.7 & 28.2 & 25.3 & 9.0 & 13.1 & 24.1 & 2.3 & 0.62 & $* *$ \\
\hline Red' (R') & $625-699 \mathrm{~nm}$ & 36.8 & 34.8 & 30.3 & 57.2 & 52.1 & 19.3 & 71.8 & -0.35 & ** \\
\hline
\end{tabular}

a) By conventional wavelength band. b) The wavelength range of $425-700 \mathrm{~nm}$ was divided into five bands at intervals of 50 or $75 \mathrm{~nm}$, referring to Figure 1 and Kong et al. (2015) [13]. c) Simple correlation coefficient with shoot fresh weight $(F W)$. The R's with ${ }^{* *}$ are statistically significant at $\mathrm{p}=0.01$. The critical correlation coefficient at $\mathrm{p}=0.01$ for $\mathrm{n}=105$ is \pm 0.25 and that at $\mathrm{p}=0.05$ is \pm 0.19 .

this study, therefore, we confined our attention to the rest of four color-bands, for which three response patterns were recognized for $F W$ s response to \%PFD by color band (Table 3 and Figure 2); linearly declining (B-band), linearly increasing (FR-band), and following the inverted-V-shaped response pattern with a maximum (G- and R-bands). This inverted- $\mathrm{V}$-shaped response corresponded to low simple correlation coefficients between $F W$ and \%PFD for G- and R-bands (Table 3). The 50-nm-wide B'- and G'-bands and the 75-nm-width R'-band shared the same response patterns as in the conventional 100-nm bands, respectively. The response patterns for these color bands were statistically significant at $\mathrm{p}<0.05$ for $\mathrm{n}=7$, in either linear or double-log linear form, except for G'-band (Table 4). The response patterns in C (cyan)- and Y (yellow)-bands were not as clear as in other bands (Figure 2), but the search for the best-fitted response function revealed that it was the inverted-V-shape pattern for $\mathrm{C}$-band and the monotonically increasing one for Y-band (Table 4).

These results gave the following observations:

Observation III: Light in B-band correlated negatively with $F W$ for the \%PFD range from $8 \%$ to $24 \%$, the correlation of which was brought about mainly by light in B'-band (425 - $475 \mathrm{~nm}$ ). Since the "coolness" of LEDs increases as \%PFD of B-band increases, higher "coolness" was associated with lower yield performance.

Observation IV: Light in R-band had the best level of \%PFD at about 50\%; 


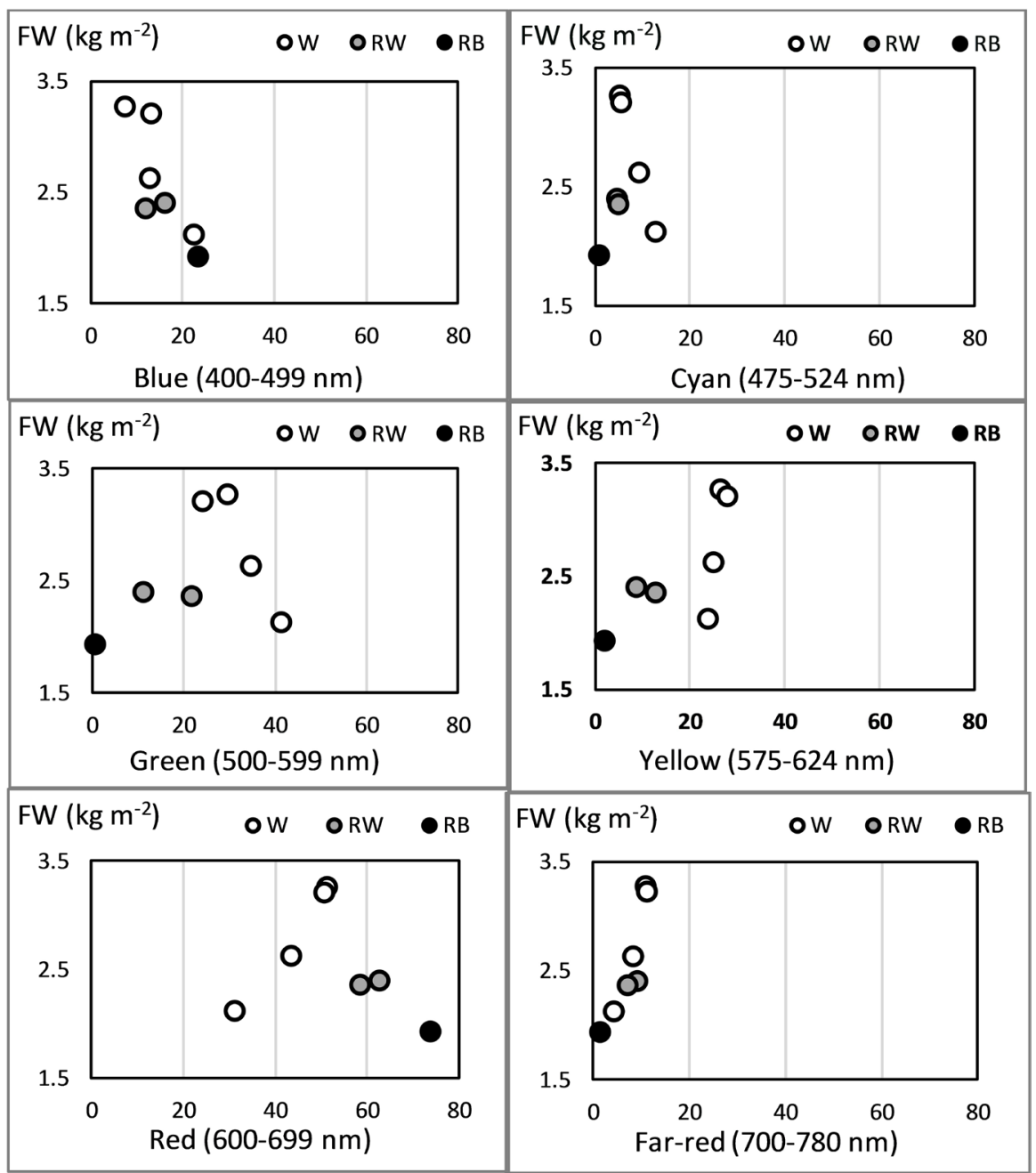

Figure 2. Response of lettuce fresh weight $(F W)$ to changes in the percentage-share of photon-flux density (\%PFD) in the total PFD for four conventional color bands and two sub-color bands (data are from Table 3).

before this level, $F W$ increased as red light increased, but beyond it, an increase in red light was associated with $F W$ negatively. If red light alone is used without any light in other light bands, it is hardly conceivable that increasing PFD (not \%PFD) from the 50\% lower level to the given light intensity level of 200 $\mu \mathrm{mol} \cdot \mathrm{m}^{-2} \cdot \mathrm{s}^{-1}$ gave a negative effect on plant growth. Therefore, the inverted-Vresponse in R-band, coupled with Observation III on B-band, implies that light in G- and FR-bands had positive effects on plant growth.

Observation V: The presence of light in FR-band correlated positively with $F W$ in the $\%$ PFD range from $2 \%$ to $11 \%$.

Observation VI: The lack as well as the excess of light in G-band appeared to give adverse effects on $F W$, with the best \%PFD of $30 \%$. It is apparent that the difference in the productivity between the two RW-LEDs and the RB-LED corresponded to whether green light exists or not.

Observation VII: The response curve in Y-band was of monotonically in- 
creasing one, though not statistically significant at $\mathrm{p}=0.05$ (Table 4), which suggests that the effect of yellow light on plant growth is neutral, if not positive.

Observation VIII: The high $\mathrm{R}^{2}$ s $\mathrm{s}$ in the estimation of the response function for B'-, R-, R'-, and FR-bands (Table 4) suggest that the relative PFD quantities in the entire spectrum of these bands were highly related to the yield performance of lettuce.

\section{Discussion}

The eight observations obtained from our experiment were based on graphical and simple statistical analyses, none of which assures that the associations we found are causal or deterministic relationships between spectrum characteristics and plant growth. To overcome this defect, we try to compare our results with those of past studies in order to assess how general can be our observations about the effects of whitish LEDs on lettuce growth. This is not an easy task, as stated in a recent review by Ouzounis et al. (2015) [6] "One of the greatest challenges for the LED as alternative light source for greenhouses and closed environments is the diversity of the way experiments are conducted that often makes results difficult to compare." A similar statement is also found in Pinho et al. (2012) [17]. The recent increase in the literature in this field makes it possible for us to challenge this difficulty. The yield performance of 10 studies, including

Table 4. The best-fitted fresh-weight response functions to the percentage share of photon flux density (\%PFD) by wavelength band $(\mathrm{n}=7)^{\mathrm{a}}$.

\begin{tabular}{ccccc}
\hline & Slope $^{\mathrm{b}}$ & $\mathrm{R}^{2}$ & Prob. $^{\mathrm{c}}$ & Response pattern; functional form $^{\mathrm{d}}$ \\
\hline Blue $(400-499 \mathrm{~nm})$ & - & 0.724 & 0.015 & Monotonic; double-log linear \\
B' $(425-474 \mathrm{~nm})$ & - & 0.908 & 0.001 & Monotonic; double-log linear \\
C $(475-524 \mathrm{~nm})$ & - & 0.584 & 0.046 & Inverted-V; double-log linear \\
Green $(500-599 \mathrm{~nm})$ & $(-)$ & 0.634 & 0.032 & Inverted-V; linear \\
G' $(525-574 \mathrm{~nm})$ & $(-)$ & 0.507 & 0.073 & Inverted-V; linear \\
Y $(575-624 \mathrm{~nm})$ & + & 0.533 & 0.063 & Monotonic; linear \\
Red $(600-699 \mathrm{~nm})$ & $(-)$ & 0.877 & 0.002 & Inverted-V; linear \\
R' $(625-699 \mathrm{~nm})$ & $(-)$ & 0.944 & $3 \mathrm{E}-04$ & Inverted V; double-log linear \\
Far red $(700-780 \mathrm{~nm})$ & + & 0.819 & 0.005 & Monotonic; linear \\
\hline
\end{tabular}

a) Flesh-weight $(F W)$ response functions (Equation (1)) is estimated using the data presented in Table 3 for seven LEDs. For the response pattern in which $F W$ increases or decreases throughout the wavelength range concerned without any maximum except for the edge points, $F W$ is regressed to \%PFD by color band. Three functional forms are tried out: i) linear, ii) semi-log linear, and iii) double-log linear, and the best-fitted form in terms of the coefficient of determination $\left(\mathrm{R}^{2}\right)$ is selected. In case $F W$ follows an inverted-V distribution with the maximum, $F W$ is regressed to the absolute value of the deviation from the best \%PFD (i.e., the \%PFD of LED\#1) for each of seven LEDs. For this pattern, too, three linear models are tried out. b) The sign of the estimated slope parameter ( $b$ in Equation (1)). For the inverted-V shape response pattern, this parameter must be negative by definition. c) The probability that the null hypothesis of the slope coefficient $=0$ is accepted, or equivalently, the null hypothesis of no correlation between $F W$ and $\mathrm{X}$ in Equation (1) is accepted, for $\mathrm{n}=7$. d) The response pattern and the functional form of the best-fitted linear model. 
ours, in which lettuce was grown under perfectly controlled closed-system conditions using whitish LEDs, are summarized in Table 5, together with spectral distribution among the color bands for LEDs used.

\subsection{Yield Performance}

Yield performance is of primary importance for plant factory entrepreneurs. The range of lettuce yields and mean yields in our experiment were comparable with the levels of yield reported by previous studies. A large number of experiments in this field revealed that the yield of leaf lettuce in closed plant factory systems

Table 5. Ranking of light sources in terms of the yield of lettuce, grown under closed-system conditions for a given light intensity, reported by recent studies with whitish LEDs.

\begin{tabular}{|c|c|c|c|}
\hline Data source & $\begin{array}{l}\text { Ranking }^{\mathrm{a}} \\
\text { Light sources are ordered according to the mean shoot fresh (or dry) weights from the top to } \\
\text { the bottom, and the mean difference between sources followed by the same lower-case } \\
\text { bold-face letter is not statistically significant at } \mathrm{p}<0.05 \text {. }\end{array}$ & $\begin{array}{l}\operatorname{Max} F W \\
(\text { g.plant } \\
\end{array}$ & $\begin{array}{c}\text { Total PFD } \\
\left(\mu \mathrm{mol} \cdot \mathrm{m}^{-2} \cdot \mathrm{s}^{-1}\right)\end{array}$ \\
\hline $\begin{array}{l}\text { Lin et al. } \\
(2013)[10]^{\mathrm{b}}\end{array}$ & $\mathrm{RB}+\mathrm{W} \mathbf{a} ; \mathrm{RB} \mathbf{b} ; \mathrm{FL} \mathbf{b}$ (warm) & 164.1 & 210 \\
\hline Han et al. (2017) $[8]^{\mathrm{b}}$ & WW a; RB ab (low B/R); R ab; CW b (high B/R); CW bc (low B/R); B cd; RB d (high B/R) & 66.0 & 150 \\
\hline $\begin{array}{l}\text { Zhang et al. (2018) } \\
{[12]^{\mathrm{b}}}\end{array}$ & $\mathrm{R}$ a; RB ab (B: R = 20:80); RB ab (10:90); B ab; P ab; W bc (type unspecified); Y c; G d & 61.0 & 200 \\
\hline Park et al. (2012) [15] & RW a (B:G:R:FR = 18:9:71:1); CW a (25:51:21:2); FL b $(30: 44: 25: 1)$ & 26.4 & 140 \\
\hline Bian et al. (2016) [11] & RB a (B:G:R = 20:0:80); CW b $(42: 42: 16)$ & 26.4 & 200 \\
\hline Cope et al. (2014) [7] ${ }^{\mathrm{c}}$ & $\begin{array}{l}\text { NW a (B:G:R:FR = 18:43:33:6); RGB a }(14: 23: 63: 0) ; \text { WW ab }(10: 37: 44: 9) ; \text { CW b }(26: 46: 24: 4) \\
\text { RB b (12:2:86:0); B bc }(92: 8: 0: 0) ; \text { R c }(0: 2: 98: 0)\end{array}$ & $(0.36)$ & 200 \\
\hline $\begin{array}{l}\text { Hytönen et al. (2017) } \\
\text { [9] }\end{array}$ & $\begin{array}{l}\text { WW a (B:G:R:FR = 11:32:48:10); HPS a }(5: 42: 43: 10) ; \text { WW a }(7: 33: 50: 10) ; \text { RB b }(18: 0: 81: 1) ; \text { RW } \\
\text { b }(17: 26: 54: 2)\end{array}$ & 117.8 & $128-144$ \\
\hline $\begin{array}{l}\text { Mickens et al. (2018) } \\
{[14]}\end{array}$ & $\begin{array}{l}\text { RGB + FR a (B:G:R:FR = 14:20:50:16); NW+R ab (16:38:46:0); NW+FR bc }(17: 41: 27: 15) \\
\text { NW + G bc }(17: 58: 25: 0) ; \text { NW cd }(20: 47: 32: 0) ; N W+B \text { de }(43: 36: 23: 0) ; \text { RB e }(40: 0: 60: 0)\end{array}$ & 30.1 & 180 \\
\hline \multirow[t]{2}{*}{$\begin{array}{l}\text { Kong et al. (2015) } \\
{[13]^{\mathrm{d}}}\end{array}$} & $\begin{array}{l}\text { RGB a (B:G:R = 26:23:51); CW ab (28:51:21); RB ab (21:0:79); RGB ab (26:40:34); FL bc } \\
(12: 30: 58) ; \text { FL c (36:40:23) } \\
\text { RGB a (B':C:G':Y:R' = 19:16:12:2:51); CW ab (24:9:31:24:12); RB ab (16:4:0:8:72); RGB ab } \\
(18: 26: 19: 8: 29) ; \text { FL bc (7:6:20:33:34); FL c }(25: 15: 27: 25: 8)\end{array}$ & 90.9 & 150 \\
\hline & WW a (B:G:R:FR = 8:30:51:11); WW a (13:24:51:11); WW b (13:35:44:8); RW b (16:11:63:9); & 97.9 & 200 \\
\hline
\end{tabular}

Our study RW bc (12:22:59:7); NW cd (23:42:31:4); RB d (24:1:74:2)

WW a (B':C:G':Y:R':FR = 6:5:14:27:37:11); WW a (7:6:9:28:35:11); WW b $(9: 10: 17: 25: 30: 8)$; RW b (13:5:6:9:57:9); RW bc (10:5:12:52:7); NW cd (17:13:21:24:19:4); RB d (22:1:0:2:72:2)

a) Light sources are abbreviated as follow: White-LED = blue-LED + phosphor $=\mathrm{W}$, Narrow-spectrum LED $[\mathrm{B}=\mathrm{blue-LED}, \mathrm{G}=$ green-LED), $\mathrm{P}=$ purple-LED, R = red-LED, Y = yellow-LED, RB = red + blue-LED], Broad-spectrum LED [CW = cool white-LED, NW = neutral white-LED, WW = warm white-LED, RW = red + white-LED, RGB = red + green + blue-LED]; Other lights [FL = fluorescent light, HPS = high pressure sodium light]. Such a light source as NW+FR means that a NW-LED is supplemented by a FR-LED. For each light source, its spectrum composition is given in parenthesis in terms of \%PFD by wavelength-band. Except for the last two studies in the list, the wavelength bands are the ones conventionally adopted: B (400 - 500 nm), G (500 - $600 \mathrm{~nm}), \mathrm{R}(600-700 \mathrm{~nm})$, and FR (700 nm and above). b) No numerical information on the spectrum characteristics was reported. c) Reports the dry weight (DW) of lettuce, instead of $F W$, which is shown in parenthesis in the yield column. d) This study divided the photosynthetic spectrum into the following five color bands: B' (400 - 475), C (475 - 525), G' (525 - 575), Y (575 - 625), and R' (625 - 700). For the purpose of comparison with other studies, the spectrum distribution among the conventional color bands are also shown for the LEDs used in their experiment. e) The spectrum distribution among the narrowly divided color bands with C- and Y-bands is shown for the purpose of comparison with Kong et al. (2015) [13]. 
ranges from $2 \mathrm{~kg} \cdot \mathrm{m}^{-2}$ to $3 \mathrm{~kg} \cdot \mathrm{m}^{-2}$ [18]. This range nearly exactly coincides with the range of $F W$ in this study (Table 2). Among the past studies, the best lettuce yields among light treatments tested ranges from $26.4 \mathrm{~g} \cdot \mathrm{plant}^{-1}$ to $164 \mathrm{~g} \cdot \mathrm{plant}^{-1}$ (Table 5). The highest lettuce yield in our study, $97.9 \mathrm{~g} \cdot$ plant $^{-1}$, belonged to a high productivity group.

\subsection{Warm-LED versus Cool-LED, Whitish-LED versus RB-LED}

The results of our experiment showed that broad-spectrum whitish-LEDs performed better than the narrow-spectrum RB-LED (Observation I), and that, among whitish-LEDs, warmer-LEDs outperformed cooler ones (Observation II). How can these findings be generalized?

At a glance, the results of the past studies seem to be diverse. In eight out of 10 studies, whitish-LEDs perform best, but the results of two studies are contradictory to Observation I. Bian et al. (2016) [11] showed that a narrow-spectrum RB-LED performed significantly better than a broad-spectrum CW-LED and Zhang et al. (2018) [12] reported that a monochromatic R-LED performed significantly better than a W-LED. Among whitish-LEDs, Park et al. (2012) [15] showed that an RW-LED performed significantly better than a CW-LED, Mickens et al. (2018) [14] reported that an RGB-LED significantly outperformed many W-LEDs, and Kong et al. (2015) [13] found no significant difference in the lettuce yield among RGB-, W-, and RB-LEDs. Does such diversity make it difficult to compare these studies in search of general understanding about how $\mathrm{W}$-LEDs affect the yield performance?

Our Observation III tells that the yield performance declines as \%PFD in B-band, which determines the coolness of LEDs, increases. Among the lights of different colors, the effects of blue light on plant growth have been well established, next to those of red light. As reported in a pioneering study [19], an increase in blue light facilitates plant growth at very low levels of \%PFD $(<16 \%)$, but its further increases give negative effects on it [7] [20]. In the most detailed study on the effects of blue and red lights on plant growth using narrow-spectrum, high-peak LEDs [21], it was shown that the dry weight of lettuce declined monotonically as blue light increased, over 11 treatments for $\% \mathrm{PFD}$, from $13 \%$ to $100 \%$. Since the response curve of red light is the reverse of that of blue light emitted by a narrow-spectrum RB-LED, the lettuce yield increased monotonically as red light increased from $0 \%$ to $87 \%$ PFD and remained at the same level for the range of $87 \%-100 \%$ PFD [21]. For a given light intensity at the surface of cultivation panels, plant growth is facilitated as the "warmness" of LEDs increases, until the turning point is reached for any light source including red and blue.

Our Observation IV suggests that these turning points come at much lower levels of \%PFD if white light is included. This observation is supported by many studies, with as well as without whitish LEDs. It was found, in experiments using FLs and RB-LEDs [20] [22], that these turning points were $15 \%$ \& $61 \%$ [20] and 
$10 \% \& 44 \%$ [22] for the blue- \& the red-bands, respectively. Among the studies with whitish LEDs in Table 5, the combination of these turning points were found at 18\% \& 33\% (Cope et al. 2014) [7], 11\% \& 48\% (Hytönen et al. 2017) [9], 14\% \& 50\% (Mickens et al. 2018) [14], 26\% \& 51\% (Kong et al. 2015) [13], and $8 \%$ \& $51 \%$ (our study), all with respect to the conventional B- and R-bands. Although these turning points at which the maximum lettuce yield was attained differ depending, among others, on the \%PFD of G- and FR-bands, the differences were not large, indicating that, for broad-spectrum LEDs, the best yield performance was attained when 5\% to $25 \% \mathrm{PFD}$ and around 50\%PFD were distributed to B- and R-band, respectively.

These observations on the effects of blue and red lights on plant growth explain much of the diversity in the past studies. The yield performance of whitish-LEDs depends on their "warmness": a small amount of blue light facilitates plant growth, but the amount of red light must be more than blue light. This means the performance among the three types of W-LED is expected to be $\mathrm{WW}>\mathrm{NW}>\mathrm{CW}$, and this ordering was not violated in the four studies which used more than two types of W-LED (Table 5). In Cope et al. (2014) [7], the top mean yield was attained by a NW-LED, but it was not statistically different from the mean yields of W-LEDs of other types. Six studies in Table 5, which tested W-LEDs together with either RW-LEDs or RGB-LEDs, showed that the yield performance did not differ significantly among three types of whitish-LEDs with the similar levels of "coolness"/ "warmness" or the similar \%PFD compositions for B- and R-bands, though there were a few exceptions. The comparison between broad-spectrum whitish-LEDs and narrow-spectrum RB-LEDs or monochromatic LEDs in the past studies in Table 5 reveals that broad-spectrum LEDs perform significantly better than narrow-spectrum LEDs, unless the former LEDs were too "cool". The high \%PFD of B-band and the low \%PFD of R-band of the CW-LED used by Bian et al. (2016) [11] explain the better performance of RB-LED over CW-LED. The study by Zhang et al. (2018) [12], in which an R-LED performed better than a W-LED, the type of the W-LED is not specified.

\subsection{Effects of Lights in Non-Blue and Non-Red Wavelength Bands of Whitish-LEDs}

The results of our study showed that the \%PFD of FR-band was highly correlated with $F W$ positively, suggesting that FR light may be critical for plant growth, in addition to red and blue lights.

FR light is known to have no direct effect on photosynthesis while a decrease in red/far-red ratio enhances photosynthetic activity by changing morphological features of leaves or stems, resulting in an increase in crop yield [5] [6]. Statistical supports for this contention have been mixed. It was found in an experiment, in which W-FLs were supplemented by one of UV, B, G, R, and FR lights, that FR light was the only light that increased the yield of lettuce [23]. It was also 
found in an experiment using RB- and FR-LEDs that increases in the \%PFD of FR-band from $0.4 \%$ to $12 \%$ (four treatments) increased lettuce $F W$ significantly [24]. However, the results of another study using R- and FR-LEDs showed no systematic effect on lettuce $F W$ for five levels of \%PFD in FR-band from $0 \%$ to $50 \%$; neither monotonic nor inverted- $\mathrm{V}$ response [25]. In addition to ours, four studies with whitish-LEDs in Table 5 included light in FR-band in their experiments. Of these studies, Hytönen et al. (2017) [9] found highly significant positive effects of the \%PFD in FR-band on FW; Park et al. (2012) [15] and Micken et al. (2018) [14] found similar positive effects, but not statistically significant; and Cope et al. (2014) [7] found no systematic relation between them. It is generally the case that a small quantity of FR-light gives a positive effect on plant growth.

The positive effects of green light have been recognized recently [6]. Of particular importance for plant growth is the characteristic of green light to penetrate through the complex leaf-canopy system to leaves at lower layers near the ground surface; in contrast, blue and red lights are absorbed by leaves at top and upper layers of the canopy [26]. This ability of green photons to fine-tune whole-canopy efficiency is essential for crops such as lettuce, which have shade-making leaf-canopy systems. This contention was first demonstrated by Kim et al. (2004) [20] using RB-LEDs and FLs, the data of which showed a clear inverted-V response curve for G-band with the best \%PFD of $24 \%$. Our study reproduced nearly the same response curve, and so were other four studies in Table 5. The results of these studies support our Observation VI: a small amount of green light helps plant growth, but its excess is associated negatively with $F W$. Other two studies in Table 5 are exceptional in this respect. The number of light treatments of Park et al. (2012) [15] is too small to detect a systematic response pattern. Bian et al. (2016) [11] offers an interesting case: an RB-LED with no green light outperformed significantly a CW-LED with $42 \% \mathrm{PFD}$ in each of G- and B-bands, leaving only 16\%PFD in R-band (Table 5). Above mentioned Kim et al. (2004) [20] gave the same case with the following statistically significant ranking for lettuce $F W:$ RB-LED + G-FL $(\mathrm{B}: \mathrm{G}: \mathrm{R}=15: 24: 61)>$ CW-FL (19:51:30) = RB-LEDs (16:0:84) > G-FL (10:86:4), where "=" means no statistically significant difference in $F W$ between the two LEDs. These results imply that the negative effects of "coolness" on plant growth outweighs the positive effects of green light, if any.

A salient feature of WW-LEDs used in our experiment was the heavy presence of broad-spectrum yellow light as compared to the RB-LED and RW-LEDs which were with no or very little yellow light (Figure 1, Table 3). Yellow light has been least studied color in the research of artificial lighting for plant production. A recent review in 2017 [26] cites only one paper, Dougher and Bugbee (2001) [27], as presenting the evidence of a suppressive effect of yellow light (580 - $600 \mathrm{~nm}$ ) on lettuce growth. Although negatively-sloped dry matter (DM) response functions for yellow light are their evidence, close scrutiny of their data 
reveals that this negative correlation critically hinges on the two observations which give nearly null DWs (0.01 and $\left.0.04 \mathrm{~g} \cdot \mathrm{plant}^{-1}\right)$ under the same light treatment with \%PFDs of B:G:Y:R:FR = 0.1:31:29:30:10. If the negative response of DW to yellow light is taken as granted, DW declines to nearly null when \%PFD in Y-band increases to $29 \%$. If the inverted-V-shaped response of DW to blue light is taken as granted, the nearly null DW is due to the lack of blue light. The question is which is more decisive in determining the nearly null DW. Recently, Kong et al. (2015) [13] found that among five color bands, green light (525 - 575 $\mathrm{nm}$ ) gave the highest positive contribution to lettuce $F W$, while yellow light (575 - $625 \mathrm{~nm}$ ) had no contribution (0\%). No contribution means no suppressive, no inhibitive effect, and, in fact, their CW-LED with a relatively high Y-band \%PFD of $24 \%$ attained a $F W$ level not significantly different from the highest one (Table 5).

\section{Conclusions}

Our study showed that for a given light intensity at the surface of cultivation panels, broad-spectrum whitish-LEDs perform better than narrow-spectrum RB-LEDs in plant factory lettuce production. Among whitish-LEDs, lettuce yield performance depends on the "coolness" or "warmness" of the LEDs, or the balance of the quantity of lights between the blue- and the red-bands; not too "cool" whitish-LEDs perform better. In addition, the presence of certain amount of green and far-red light helps plant growth. Altogether, broad-spectrum whitish-LEDs outperform narrow-spectrum high-peaked RB-LEDs. The best balance of lights in different color bands certainly varies depending on many factors related to growth conditions, such as temperature, $\mathrm{CO}_{2}$ concentration, and nutrient solution, but recent studies, including ours, suggest that the preferable spectrum distribution for whitish-LEDs to attain better performance in plant growth would be, in terms of \%PFD by conventional color band, as follows: $0 \%$ $<$ blue $<30 \%$; $0 \%<$ green $<50 \% ; 30 \%<$ red $<70 \%$; and $0 \%<$ far-red $<20 \%$.

These findings have a straightforward implication for plant factory entrepreneurs in selecting LEDs for plant production; select W-LEDs of appropriate spectrum characteristics. Of the three types of whitish-LEDs, i.e., W-LEDs, RW-LEDs, and RGB-LEDs, W-LEDs are the ones which will be flooding in the LED market to satisfy the huge demands for lighting in all spheres of human life with rapidly declining prices. Even under the price structure in 2017, it was evidently economically viable for plant factory operators to make investments for switching from conventional light sources to W-LEDs in lettuce production [28]. RW-LEDs, RGB-LEDs, narrow-spectrum RB-LEDs, and other monochromatic LEDs will remain in use in plant factory production, not as major light sources but for specific purposes, such as facilitating the concentration of certain plant nutrients, for certain crops.

Considering the enormous diversity in W-LEDs now available to plant factory entrepreneurs, it is necessary to identify more precisely the spectrum patterns of 
W-LEDs that best fit specific crops, photosynthetically as well as photo-morphogenetically. To screen out W-LEDs better suited for plant production, it is necessary to clarify how a whole spectrum affects plants. Light of a certain color band, which is ineffective for plant growth, may give different effects, if mixed with lights in other color bands. There are many unknowns about how different lights in a broad spectrum work complementary or interfere with each other in plant production. Different strategies, different from the conventional ones that have been adopted in this field of study, will be needed to identify what kind of spectral distribution is best suited for W-LEDs to be used in plant production.

\section{Conflicts of Interest}

The authors declare no conflicts of interest regarding the publication of this paper.

\section{References}

[1] Pinstrup-Andersen, P. (2018) Is it Time to Take Vertical Indoor Farming Seriously? Global Food Security, 17, 233-235. https://doi.org/10.1016/j.gfs.2017.09.002

[2] Massa, G.D., Kim, H., Wheeler, R.M. and Mitchell, C.A. (2008) Plant Productivity in Response to LED Lighting. HortScience, 43, 1951-1956. https://doi.org/10.21273/HORTSCI.43.7.1951

[3] Kozai T. (2016) Why LED Lighting for Urban Agriculture? In: Kozai, T., Fujiwara, K. and Runkle, E.S., Eds., LED Lighting for Urban Agriculture, Springer, Singapore, 4-10. https://doi.org/10.1007/978-981-10-1848-0

[4] Cho, J., Park, J., Kim, J. and Schubert, E.F. (2017) White Light-Emitting Diodes: History, Progress, and Future. Laser Photonics Reviews, 11, Article ID: 1600147. https://doi.org/10.1002/lpor.201600147

[5] Goto, E. (2003) Effects of Light Quality on Growth of Crop Plants under Artificial Lighting. Environment Control in Biology, 41, 121-132. https://doi.org/10.2525/ecb1963.41.121

[6] Ouzounis, T., Rosenqvist, E. and Ottosen, C.O. (2015) Spectral Effects of Artificial Light on Plant Physiology and Secondary Metabolism: A Review. HortScience, 50, 1128-1135. https://doi.org/10.21273/HORTSCI.50.8.1128

[7] Cope, K.R., Snowden, M.C. and Bugbee, B. (2014) Photobiological Interactions of Blue Light and Photosynthetic Photon Flux: Effects of Monochromatic and BroadSpectrum Light Sources. Photochemistry and Photobiology, 90, 574-584. https://doi.org/10.1111/php.12233

[8] Han, T., Vaganov, V., Cao, S., Li, Q., Ling, L., Cheng, X., Peng, L., Zhang, C., Yakovlev, A.N., Zhong, Y. and Tu, M. (2017) Improving "Color Rendering” of LED Lighting for the Growth of Lettuce. Scientific Reports, 7, Article No. 45944. https://doi.org/10.1038/srep45944

[9] Hytönen, T., Pinho, P., Rantanen, M., Kariluoto, S., Lampi, A., Edelmann, M., Joensuu, K., Kauste, K., Mouhu, K., Piironen, V., Halonen, L. and Elomaa, P. (2017) Effects of LED Light Spectra on Lettuce Growth and Nutritional Composition. Lighting Research \& Technology, 50, 880-893.

[10] Lin, K., Huang, M, Huang, W, Hsu, M., Yang, Z. and Yang, C. (2013) The Effects of Red, Blue, and White Light-Emitting Diodes on the Growth, Development, and 
Edible Quality of Hydroponically Grown Lettuce (Lactuca sativa L. var. capitata). Scientia Horticulturae, 150, 86-91. https://doi.org/10.1016/j.scienta.2012.10.002

[11] Bian, Z.H., Cheng, R.F., Yang, Q.C., Wang, J. and Lu, C. (2016) Continuous Light from Red, Blue, and Green Light-Emitting Diodes Reduces Nitrate Content and Enhances Phytochemical Concentrations and Antioxidant Capacity in Lettuce. Journal of the American Society for Horticultural Science, 141, 186-195. https://doi.org/10.21273/JASHS.141.2.186

[12] Zhang, T., Shi, Y., Piao, F. and Sun, Z. (2018) Effects of Different LED Sources on the Growth and Nitrogen Metabolism of Lettuce. Plant Cell, Tissue and Organ Culture, 134, 231-240. https://doi.org/10.1007/s11240-018-1415-8

[13] Kong, S., Chung, H., Chang, M. and Fang, W. (2015) The Contribution of Different Spectral Sections to Increase Fresh Weight of Boston Lettuce. HortScience, 50, 1006-1010. https://doi.org/10.21273/HORTSCI.50.7.1006

[14] Mickens, M.A., Skoog, E.J., Reese, L.E., Barnwell, P.L., Spencer, L.E., Massa, G.D. and Wheeler, R.M. (2018) A Strategic Approach for Investigating Light Recipes for “Outredgeous” Red Romaine Lettuce Using White and Monochromatic LEDs. Life Science in Space Research, 19, 53-62. https://doi.org/10.1016/j.lssr.2018.09.003

[15] Park, Y.G., Park, J.E., Hwang, S.J., and Jeong, B.R. (2012) Light Source and $\mathrm{CO}_{2}$ Concentration Affect Growth and Anthocyanin Content of Lettuce under Controlled Environment. Horticulture, Environment, and Biotechnology, 53, 460-466. https://doi.org/10.1007/s13580-012-0821-9

[16] Murphy, K.R., Myors, B. and Wolach, A. (2014) Statistical Power Analysis: A Simple and General Model for Traditional and Modern Hypothesis Tests. 4th Edition, Routledge, New York. https://doi.org/10.4324/9781315773155

[17] Pinho, P., Jokinen, K. and Halonen L. (2012) Horticultural Lighting-Present and Future Challenges. Lighting Research \& Technology, 44, 427-437. https://doi.org/10.1177/1477153511424986

[18] Kozai T. and Niu, G. (2016) Role of the Plant Factory with Artificial Lighting (PFAL) in Urban Areas. In: Kozai, T., Niu, G. and Takagaki, M., Eds., Plant Factory: An Indoor Vertical Farming System for Efficient Quality Food Production, Academic Press, London, 7-33. https://doi.org/10.1016/B978-0-12-801775-3.00002-0

[19] Yorio, N., Goins, G.D., Kagie, H.R., Wheeler, R.M. and Sager, J.C. (2001) Improving Spinach, Radish, and Lettuce Growth under Red Light-Emitting Diodes (LEDs) with Blue Light Supplementation. HortScience, 36, 380-383. https://doi.org/10.21273/HORTSCI.36.2.380

[20] Kim, H.H., Goins, G.D., Wheeler, R.M. and Sager, J.C. (2004) Green-Light Supplementation for Enhanced Lettuce Growth under Red- and Blue-Light Emitting Diodes. HortScience, 39, 1617-1622. https://doi.org/10.21273/HORTSCI.39.7.1617

[21] Son, K.H., Lee, J.H., Oh, Y., Kim, D., Oh, M.M. and In, B.C. (2017) Growth and Bioactive Compound Synthesis in Cultivated Lettuce Subject to Light-Quality Changes. HortScience, 2, 584-591. https://doi.org/10.21273/HORTSCI11592-16

[22] Ohashi-Kaneko, K., Takase, M., Kon, N., Fujiwara, K. and Kurata, K. (2007) Effect of Light Quality on Growth and Vegetative Quality in Leaf Lettuce, Spinach and Komatsuna. Environment Control in Biology, 45, 189-198. https://doi.org/10.2525/ecb.45.189

[23] Li, Q. and Kubota, C. (2009) Effects of Supplemental Light Quality on Growth and Phytochemicals of Baby Leaf Lettuce. Environmental and Experimental Botany, 67, 59-64. https://doi.org/10.1016/j.envexpbot.2009.06.011 
[24] Pinho, P., Jokinen, K. and Halonen, L. (2017) The Influence of the LED Light Spectrum on the Growth and Nutrient Uptake of Hydroponically Grown Lettuce. Lighting Research \& Technology, 49, 866-881. https://doi.org/10.1177/1477153516642269

[25] Lee, M.J., Park, S.Y. and Oh, M.M. (2015) Growth and Cell Division of Lettuce Plants under Various Ratios of Red to Far-Red Light-Emitting Diodes. Horticulture, Environment, and Biotechnology, 56, 186-194. https://doi.org/10.1007/s13580-015-0130-1

[26] Smith, H.L., McAusland, L. and Murchie, E.H. (2017) Don't Ignore the Green Light: Exploring Diverse Roles in Plant Processes. Journal of Experimental Botany, 68, 2099-2110. https://doi.org/10.1093/jxb/erx098

[27] Dougher, T.A.O. and Bugbee, B. (2001) Evidence for Yellow Light Suppression of Lettuce Growth. Photochemistry and Photobiology, 73, 208-212. https://doi.org/10.1562/0031-8655(2001)073<0208:EFYLSO>2.0.CO;2

[28] Saengtharatip, S., Lu, N., Takagaki, M., Kikuchi, M. (2018) Productivity and Cost Performance of Lettuce Production in Plant Factory Using Various Light-EmittingDiodes of Different Spectra. ISSAAS Journal, 24, 1-9. 